\title{
HIV-1 latent reservoir size and diversity are stable following brief treatment interruption
}

\author{
D. Brenda Salantes, ${ }^{1}$ Yu Zheng, ${ }^{2}$ Felicity Mampe, ${ }^{1}$ Tuhina Srivastava, ${ }^{1}$ Subul Beg, ${ }^{3}$ Jun Lai, ${ }^{3}$ Jonathan Z. Li, ${ }^{2}$ Randall L. Tressler, ${ }^{4}$ \\ Richard A. Koup, ${ }^{5}$ James Hoxie, ${ }^{1}$ Mohamed Abdel-Mohsen, ${ }^{6}$ Scott Sherrill-Mix, ${ }^{1}$ Kevin McCormick, ${ }^{1}$ E. Turner Overton, ${ }^{7}$ \\ Frederic D. Bushman, ${ }^{1}$ Gerald H. Learn, ${ }^{1}$ Robert F. Siliciano, ${ }^{3,8}$ Janet M. Siliciano, ${ }^{3}$ Pablo Tebas, ${ }^{1}$ and Katharine J. Bar ${ }^{1}$ \\ 'University of Pennsylvania, Philadelphia, Pennsylvania, USA. ${ }^{2}$ Harvard University, Cambridge, Massachusetts, USA. ${ }^{3}$ ohns Hopkins University School of Medicine, Baltimore, Maryland, USA. ${ }^{4}$ Division of \\ AIDS (DAIDS), National Institute of Allergy and Infectious Diseases (NIAID), NIH, Bethesda, Maryland, USA. ${ }^{5}$ Vaccine Research Center, NIAID, Bethesda, Maryland, USA. ${ }^{6}$ Wistar Institute, Philadelphia, \\ Pennsylvania, USA. University of Alabama at Birmingham, Birmingham, Alabama, USA. ${ }^{8}$ Howard Hughes Medical Institute, The Johns Hopkins University School of Medicine, Baltimore, Maryland, USA.
}

BACKGROUND. The effect of a brief analytical treatment interruption (ATI) on the HIV-1 latent reservoir of individuals who initiate antiretroviral therapy (ART) during chronic infection is unknown.

\begin{abstract}
METHODS. We evaluated the impact of transient viremia on the latent reservoir in participants who underwent an ATI and at least 6 months of subsequent viral suppression in a clinical trial testing the effect of passive infusion of the broadly neutralizing Ab VRC01 during ATI.

RESULTS. Measures of total HIV-1 DNA, cell-associated RNA, and infectious units per million cells (IUPM) (measured by quantitative viral outgrowth assay [QVOA]) were not statistically different before or after ATI. Phylogenetic analyses of HIV-1 env sequences from QVOA and proviral DNA demonstrated little change in the composition of the virus populations comprising the pre- and post-ATI reservoir. Expanded clones were common in both QVOA and proviral DNA sequences. The frequency of clonal populations differed significantly between QVOA viruses, proviral DNA sequences, and the viruses that reactivated in vivo.
\end{abstract}

CONCLUSIONS. The results indicate that transient viremia from ATI does not substantially alter measures of the latent reservoir, that clonal expansion is prevalent within the latent reservoir, and that characterization of latent viruses that can reactivate in vivo remains challenging.

TRIAL REGISTRATION. ClinicalTrials.gov NCT02463227

FUNDING. Funding was provided by the NIH.

\section{Introduction}

Combination antiretroviral therapy (ART) can suppress HIV-1 viremia below the limit of detection in clinical assays and dramatically reduce the morbidity and mortality of HIV-1 infection. ART cannot eradicate HIV-1 from the body, however, as virus persists primarily in long-lived resting memory $\mathrm{CD}^{+} \mathrm{T}$ cells that encode but do not actively express replication-competent HIV-1 (1-3). Maintenance of suppressive ART prevents the small fraction of these latently infected cells that reactivate from rekindling ongoing virus replication $(4,5)$. Upon discontinuation of ART, reactivation leads to rapid recrudescence of systemic virus replication (6-9). The stable, persistent latent HIV-1 reservoir is the primary barrier to virus eradication and the target of curative strategies. In individuals who initiate ART during chronic infection and maintain viral suppression for years, the latent reservoir

Authorship note: PT and KJB contributed equally to this work. Conflict of interest: The authors have declared that no conflicts of interest exist. Submitted: January 29, 2018; Accepted: April 24, 2018.

Reference information: / Clin Invest. 2018;128(7):3102-3115.

https://doi.org/10.1172/JCl120194. has been shown to be highly stable, with a half-life of approximately 44 months (10-12).

The field currently lacks nonviral biomarkers of reservoir reduction or virologic control, making analytical treatment interruptions (ATIs) of ART a critical component of clinical trials evaluating HIV-1 cure strategies $(13,14)$. Although brief ATIs have been generally well tolerated, concerns remain about their safety and effects on the latent reservoir $(15,16)$. Determining whether ATIs lead to a substantial change in the size or diversity of the latent reservoir is important for clinical trials participants, as it could alter their options for future cure strategies; elucidating the impact of ATIs on the reservoir also informs our understanding of viral dynamics and the mechanistic underpinnings of HIV persistence. There are few objective data, however, regarding the effects of a brief ATI and transient viremia on the size and composition of the latent reservoir.

The AIDS Clinical Trials Group (ACTG) clinical trial A5340 tested the capacity for the broadly neutralizing antibody (bnAb) VRC01 to prevent or delay viral rebound after an ATI (17). The trial enrolled 14 participants who initiated ART in chronic infection and maintained long-standing viral suppression. VRC01 infusions 


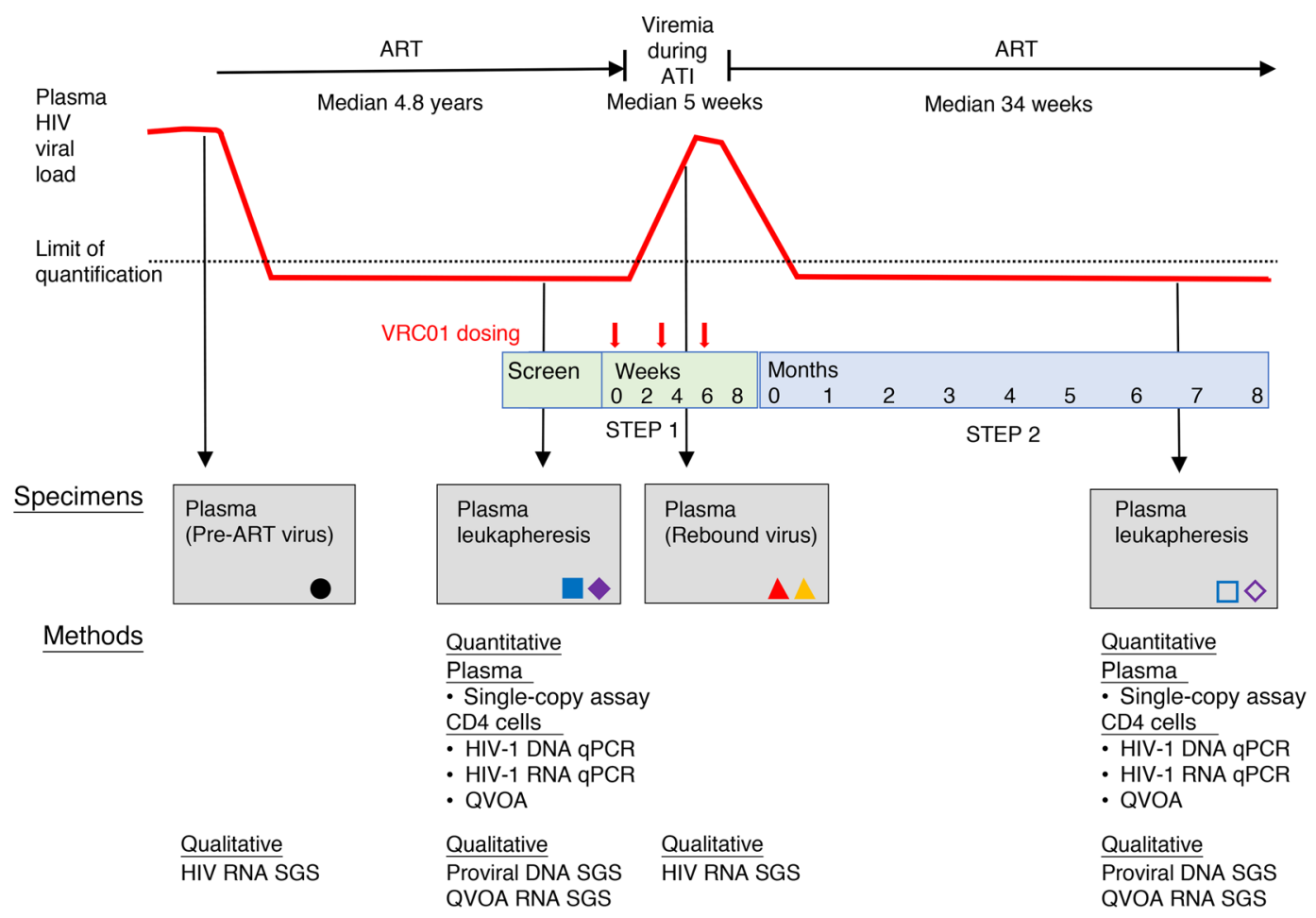

Figure 1. Study schema. The A5340 clinical trial schema is shown at the top, with the type and timing of clinical specimens obtained for the present study shown in the center, and the assays performed shown at the bottom.

were safe and well tolerated and briefly delayed viral rebound compared with historical controls, but did not lead to sustained viral suppression. Phylogenetic and phenotypic evaluation of pre-ART and rebound plasma viruses suggested that VRCO1 could restrict the clonality of viral rebound in participants with VRC01-sensitive viruses, but preexisting resistance to VRCO1 was prevalent in this cohort. After viral rebound leading to a plasma viral load of greater than 1,000 copies/ml, all participants resumed their pre-trial ART regimen and rapidly achieved viral suppression. This study provided a unique opportunity to determine the impact of transient viremia on the latent HIV-1 reservoir and to assess the relationship between samples of the HIV reservoir and rebounding virus. Using samples obtained from nine A5340 trial participants before and after ATI, we performed quantitative and qualitative analyses of the latent reservoir to determine the effect of treatment interruption and transient viremia on the size and composition of the latent reservoir. In parallel, we assessed the relatedness of the virus populations sampled from plasma and PBMCs prior to ART initiation, at study entry, at viral rebound during ATI, and after viral suppression. The results showed that ATI with transient viremia did not substantially alter the size or diversity of the peripheral reservoir. Further, clonal expansion within replication-competent and genetically intact latent virus populations was prevalent and confounded pre-trial screening approaches to identify the viruses that will reactivate in vivo upon treatment interruption.

\section{Results}

Patient population and study schema. Nine individuals participated in this component of the ACTG clinical trial A5340. As shown in the study schema (Figure 1), participants receiving infusions of VRC01 underwent ATI and were monitored weekly for viral rebound. Participants experienced viral rebound between weeks 3 and 11 of ATI and were restarted on ART after confirmation of plasma viremia above 1,000 copies/ml. We analyzed plasma and/or peripheral blood mononuclear cell (PBMC) samples from 4 time points (Figure 1): (a) Plasma samples obtained prior to ART initiation (available for 5 participants); (b) plasma obtained in the first and/or second week of viral rebound after ATI; (c) plasma and PBMCs obtained by leukapharesis upon trial entry (prior to VRC01 administration or ATI); and (d) plasma and PBMCs obtained at least 6 months after plasma viral suppression to fewer than 200 copies/ml on ART.

The participants' demographic and clinical characteristics are shown in Table 1. At study entry, all 9 participants had plasma viral loads measured below 40 copies $/ \mathrm{ml}$ and had been on suppressive ART for a median of 4.8 years (range, 3.6-14.5 years). Prior to ATI or VRC01 administration, the participants underwent leukapheresis and rectal biopsy. After ART interruption, the participants were monitored weekly for detectable viremia, and ART was reinitiated upon confirmation of a plasma viral load of more than 1,000 copies $/ \mathrm{ml}$. The median duration of viremia of more than 200 copies/ml during ATI was 5 weeks (range, 4-6 weeks). The participants' plasma viral load was resuppressed to fewer than 200 copies/ml on ART for a median of 34 weeks (range, 23-44 weeks) prior to the second leukapheresis. The specifics of each participant's time on ART prior to ATI, time to viral rebound, and peak viral load during ATI are shown in Supplemental Figure 1 (supplemental material available online with this article; https:// doi.org/10.1172/JCI120194DS1). 


\section{Table 1. Baseline, clinical, and study characteristics}

\begin{tabular}{|c|c|}
\hline \multicolumn{2}{|l|}{ Characteristic } \\
\hline Male sex, $n(\%)$ & $9(100 \%)$ \\
\hline Age (yr) at entry, median $n(\mathrm{Q} 1, \mathrm{Q} 2)$ & $43(36,47)$ \\
\hline \multicolumn{2}{|l|}{ Race, $n(\%)$} \\
\hline Black or African American & $4(44 \%)$ \\
\hline White & $5(56 \%)$ \\
\hline \multicolumn{2}{|l|}{ Ethnicity, $n$ (\%) } \\
\hline White non-Hispanic & $4(44 \%)$ \\
\hline Black non-Hispanic & $3(33 \%)$ \\
\hline Hispanic & $2(22 \%)$ \\
\hline Baseline CD4 ${ }^{+} \mathrm{T}$ cell count (cells/ml), median $n(\mathrm{Q} 1, \mathrm{Q3})$ & $874(599,1,026)$ \\
\hline \multicolumn{2}{|l|}{ Nadir CD4 ${ }^{+} \mathrm{T}$ cell count (cells/ml), $n$ (\%) } \\
\hline $201-500$ & $8(89 \%)$ \\
\hline$>500$ & $1(11 \%)$ \\
\hline Years on ART at entry, median $n$ (min, max) & $4.8(3.6,14.5)$ \\
\hline \multicolumn{2}{|l|}{ ART regimen at study entry, $n(\%)$} \\
\hline INSTI based & $6(67 \%)$ \\
\hline Pl based & $3(33 \%)$ \\
\hline Total no. of wk off ART during ATI, median $n$ (min, max) & $6(3,13)$ \\
\hline $\begin{array}{l}\text { Total no. of wk with detectable viremia (>200 copies/ml) during } \\
\text { ATI, median } n \text { (min, max) }\end{array}$ & $5(4,6)$ \\
\hline $\begin{array}{l}\text { Total no. of wk from viral suppression (<200 copies/ml) on ART } \\
\text { to post-ATI leukapharesis, median } n \text { (min, max) }\end{array}$ & $34(23,43)$ \\
\hline
\end{tabular}

Quantitative measures of reservoir change. We performed several assays to quantitatively estimate within-person changes in the size of the peripheral latent reservoir before and after ATI. Using PBMCs obtained during ART suppression of viremia prior to ATI and 6 months after viral resuppression on ART (Figure 1), we negatively selected $\mathrm{CD} 4^{+} \mathrm{T}$ cells and measured the levels of total HIV-1 DNA and cell-associated RNA by quantitative PCR (qPCR) for all participants, except participant A06, in whom DNA levels were undetectable because of primer failure (Figure 2). Total DNA levels ranged from 21.4 to $1,791.2$ copies/million $\mathrm{CD}^{+} \mathrm{T}$ cells before ATI and from 25.4 to $1,564.5$ copies/million $\mathrm{CD}^{+}{ }^{+} \mathrm{T}$ cells after ATI. We found no significant change between the within-person pre- and post-ATI values, with a median $\log _{10}$ change (first quartile [Q1], third quartile [Q3]) of $0.08(-0.13,0.18)(P=0.313)$. Likewise, we detected no significant changes in cell-associated RNA levels between pre- and post-ATI samples. RNA measures at both time points ranged from 6.1 to 10,834.7 copies/million cells, with a median $\log _{10}$ change $(\mathrm{Q} 1, \mathrm{Q} 3)$ of $-0.05(-0.15,0.2)(P=0.734)$.

Using the same PBMC samples collected before and after ATI, we measured the frequency of resting $\mathrm{CD}^{+}{ }^{+} \mathrm{T}$ cells harboring replication-competent virus using a quantitative viral outgrowth assay (QVOA) (18). Infectious units per million cells (IUPM) ranged from 0.033 to 16.248 IUPM before the trial and from 0.023 to 8.35 IUPM after the trial. We found no evidence suggesting significant within-person changes, with a median $\log _{10}$ change $(\mathrm{Q}, \mathrm{Q} 3)$ of 0.3 $(-0.16,0.63)(P=0.652$, Figure 2$)$.

We tested the association between the 3 quantitative assessments at baseline, after the trial, and in the change between time points. We found a modest correlation between the measures of total HIV-1 DNA in CD4 ${ }^{+} \mathrm{T}$ cells and replication-competent virus recovered via viral outgrowth of resting $\mathrm{CD} 4^{+} \mathrm{T}$ cells, with Spearman's correlation coefficients of 0.68 and $(P=0.06)$ at baseline, $0.83(P=0.01)$ after ATI, and $0.79(P=0.02)$ for the change between baseline and after ATI (Supplemental Figure 2).

A single-copy assay was performed to detect low-level viremia in plasma collected during suppressive ART before and after the trial (Supplemental Figure 3). With a limit of detection of fewer than 0.5 copies $/ \mathrm{ml}$, viremia in 3 participants remained undetectable before and after ATI, viremia was above detectable levels at both time points in 3 participants, the levels in 1 participant went from just above detection $(0.53$ copies $/ \mathrm{ml})$ to undetectable, while the remaining 2 participants had plasma viral loads that went from undetectable to 2.14 copies $/ \mathrm{ml}$ and 2.19 copies $/ \mathrm{ml}$, respectively.

Phylogenetic analysis of reservoir change. We next tested whether treatment interruption and transient viremia changed the composition of the viral reservoir populations using phylogenetic analysis of 8 participants. Participant A14 had a limited sequence yield and was not included in the analyses. Participant A09 was infected with both subtype A and B viruses (17); only subtype B viruses were included in these analyses. We performed single-genome sequencing (SGS) of gp160 env from virus replicating in all available $\mathrm{p} 24^{+}$culture wells of the pre- and post-ATI QVOA assays (8 participants). For each culture well, the consensus sequence of multiple SGS-derived env sequences is shown; cultures with evidence of multiple templates were excluded from analysis (see Methods). QVOA culture env sequences were compared with SGS-derived gp160 env sequences from plasma virus sampled prior to ART initiation (available for 5 participants) and plasma virus sampled during the first and second weeks of detectable rebound viremia after ATI. When analyzed together in a maximum likelihood (ML) tree, the 8 participants' sequences clustered independently (17).

Clonal populations are prevalent within the replication-competent peripheral latent reservoir. Figures 3 and 4 display ML phylogenetic trees of each individual participant's gp160 env sequences from pre-ART, rebound, and pre- and post-ATI QVOA time points. Participant sequences showed a range of maximum within-patient diversity (range, $0.87 \%-6.1 \%$ ) and tree structure, as expected for sequences obtained after ART initiation during chronic infection. For 7 of 8 participants, viral rebound was polyclonal, with multiple genetically distinct, low-diversity rebound lineages replicating systemically within weeks of ATI (17).

Upon examination of the QVOA sequences, we found multiple groups of identical $e n v$ sequences. The $e n v$ sequences were generated using primers with a clonal prediction score of 95, indicating a high likelihood that identical env sequences reflected identical whole genomes (19). While definitive proof of clonal expansion requires a demonstration of identical whole genomes with identical integration sites in viruses from different cells, modeling and empiric studies showed that multiple identical gp160 env sequences provide strong support for clonal expansion (19-22). Given the mutations that can accrue during the 14 to 21 days of ex vivo QVOA culture and the in vitro error inherent in reverse transcription PCR, we included sequences with up to 2 nucleotide polymorphisms within our definition of clonal lineages $(23,24)$. 

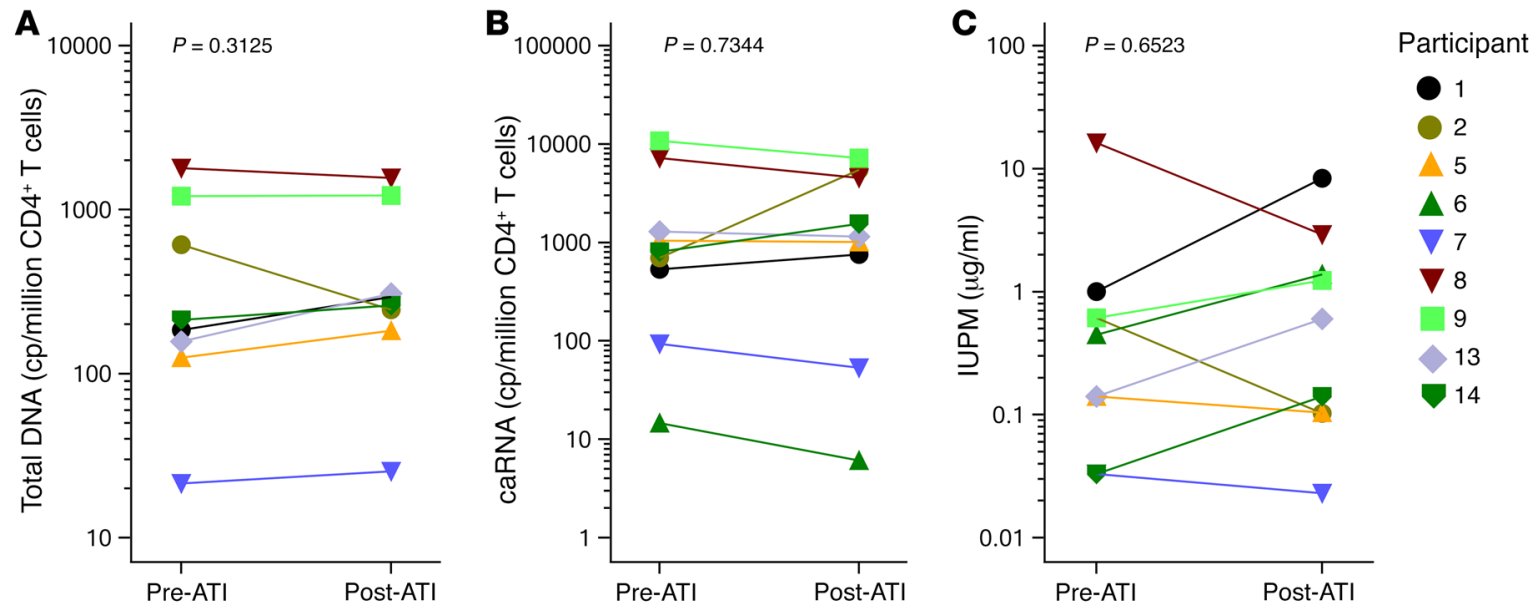

Figure 2. Quantitative measures of reservoir change. Pre-ATI and post-ATI values (obtained at study entry and more than 6 months after viral suppression following ART reinitiation, respectively) of total HIV-1 DNA in CD4+ ${ }^{+}$cells (A), cell-associated HIV-1 RNA (caRNA) in CD4 ${ }^{+}$T cells (B), and the frequency of resting CD4 ${ }^{+}$T cells bearing replication-competent virus (IUPM) (C) are shown for each A5340 participant. Total DNA is not shown for participant A06, because the values were below detectable levels at both time points. $P$ values shown in $\mathbf{A}-\mathbf{C}$ indicate the significance of the within-participant changes and were determined by Wilcoxon signed-rank test.

In each participant with sufficient (>2 per time point) QVOA sequences (A01, A02, A06, A08, A09, and A13), we found clonal sequence groups. In the 4 participants with more than $20 \mathrm{QVOA}$ sequences, clonal sequences comprised $68 \%$ of the total viruses. We note participant A01 as an extreme example of clonal expansion of replication-competent virus, with a single clonal lineage comprising 41 of 43 (95\%) of the pre- and post-trial QVOA sequences (Figure 3). Participant A02 also had a high proportion of pre-ATI QVOA virus (55\%, 6 of 11 sequences) that fell within 2 clonal lineages. Participant A08 had multiple expanded lineages, with 6 distinct groups of 2 or more identical sequences representing $48 \%$ (14 of 29) of the QVOA sequences (Figure 4).

Minimal enrichment of rebound sequences in the post-trial latent reservoir. We next looked for phylogenetic evidence that viral rebound influenced the latent reservoir. In each participant, we asked whether post-trial QVOA sequences, compared with pre-trial QVOA sequences, were enriched with rebound viruses. In 5 participants (A01, A02, A05, A09, and A13) (Figures 3 and 4), we found that no post-ATI QVOA sequences clustered with or near rebound viruses. In participant A08, in contrast, we found a suggestion of enrichment of post-trial populations with rebound sequences. The sequences for participant A08 had 2 of 6 rebound lineages that contained both rebound and QVOA sequences; a minor lineage contained sequences from both pre- and post-trial QVOAs, while the most populous rebound lineage contained 2 identical post-trial QVOA sequences.

The findings for 2 participants were more challenging to interpret phylogenetically. Participant A07 had a very small reservoir with no replication-competent virus recovered in post-trial QVOA, suggesting that the reservoir was not enhanced quantitatively, but leaving us unable to make a qualitative assessment. Participant A06 had a very low within-patient diversity (maximum of $0.83 \%$ or $\sim 15$ total nucleotide differences in env), which made distinguishing between discrete lineages challenging. The sequences for participant A06 contained a single, relatively low-diversity lineage ( $\max -$ imum difference of 3 nucleotides or $0.12 \%$ ) containing rebound, pre- and post-trial QVOA sequences. We interpret the frequencies of pre-trial QVOA sequences (70\%, 7 of 10) and post-trial QVOA sequences $(33 \%, 5$ of 15$)$ aligned with this rebound virus as suggesting the preexistence of this lineage before ATI, rather than enrichment of this lineage from rebound viremia.

Thus, in 5 of 6 evaluable participants, we found no evidence that transient viral rebound changed the composition of the reservoir.

Limited relatedness of pre-ATI QVOA to rebound sequences. We next examined the relatedness of sequences from preexisting samples (pre-ART and QVOA) to rebound viruses, asking whether the viruses recovered ex vivo in QVOA cultures are representative of those that reactivated in vivo upon treatment interruption. For all participants, the pre-trial QVOA sequences were within a few percentage points of identity with the pre-ART, rebound, and post-trial QVOA sequences and were generally interspersed within the larger phylogeny. For 5 participants (A01, A02, A05, A09, and A13), the rebound viruses did not arise from the sampled pre-trial QVOA sequences.

The lack of correlation between pre-trial QVOA and rebound virus populations is particularly notable in participants with pre-trial QVOA sequences that were composed largely of expanded clones. For example, in participant A01, 93\% (13 of 14 sequences) of pre-trial QVOA sequences belonged to a single expanded clone. Of the 4 distinct virus populations that reactivated in vivo upon ATI, none of the viruses emanated from this clonal population. In participant A02, 55\% (5 of 9 sequences) of pre-ATI QVOA sequences were part of 2 clonal populations, but no representatives of these clones reactivated during the first 2 weeks of viral rebound in vivo. Likewise, $30 \%$ (3 of 10 sequences) of the pretrial QVOA sequences for participant A09 were part of a single clone, which was not seen in the 7 distinct virus populations that rebounded in vivo. Thus, in most participants, even when pre-ATI QVOA sequences defined a latent pool with a predominant clonally expanded population, this sampling method did not specifically predict the virus that would reactivate in vivo upon ATI. 

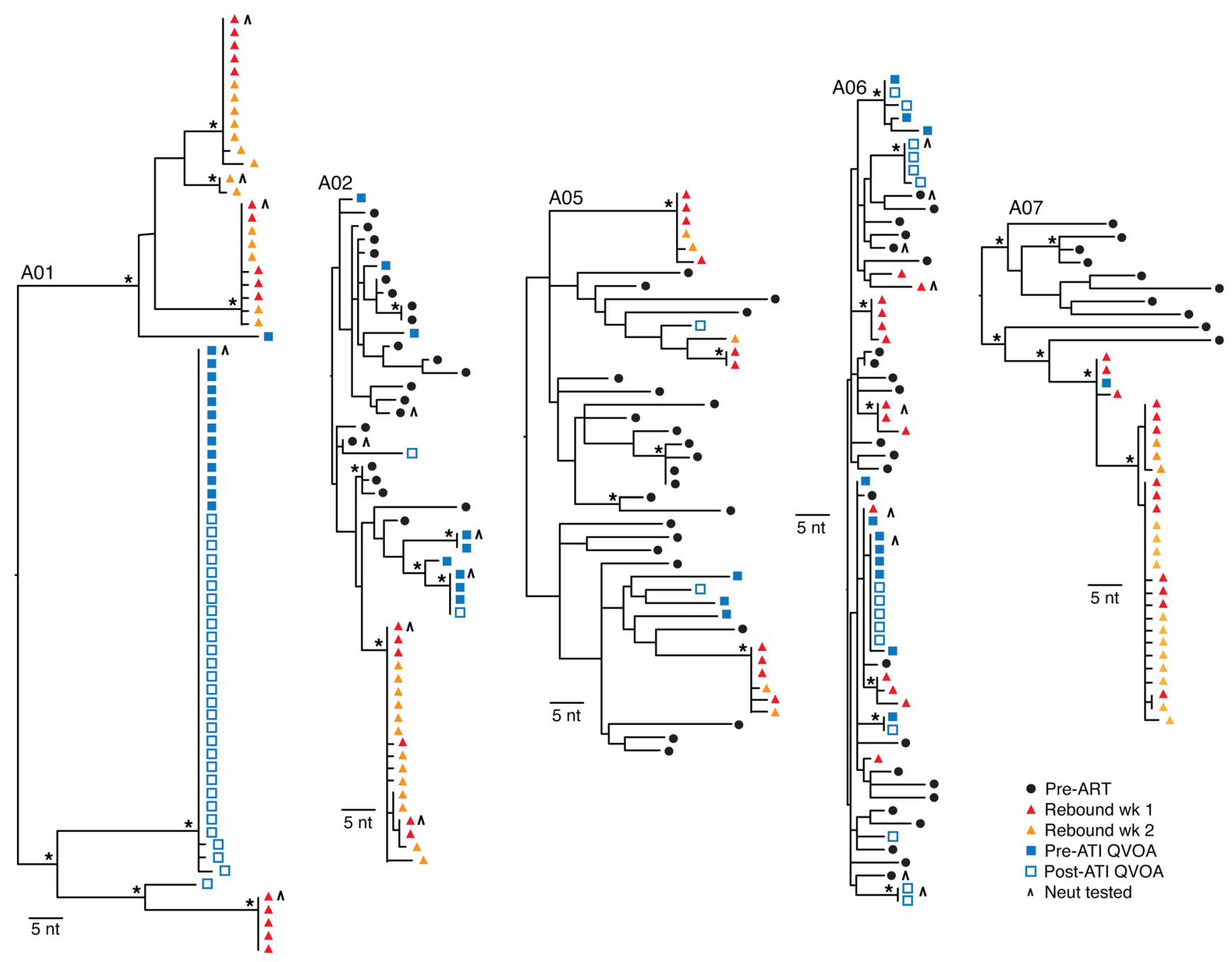

Figure 3. ML phylogenetic trees of SGS-derived gp160 env sequences from pre-ART plasma, rebound plasma, and replication-competent latent viruses for participants A01, A02, and A05-A07. Env sequences from pre-ART plasma are shown as black circles, sequences from rebound plasma from the first and second weeks of detectable viremia after ATI are shown as red and orange triangles, respectively, and sequences from virus supernatant from individual preand post-ATI p 24+ QVOA culture supernatants are shown as solid blue or open blue squares. Cenetic distance is shown by the scale bar, indicating 5 nucleotides (or $\sim 0.2 \%$ difference). Bootstrap values above 70 are indicated by asterisks. Envs tested for neutralization sensitivity (Neut tested) in participants A01, $A 02$, and $A 06$ are indicated with an inverted $v\left({ }^{\wedge}\right)$. Populations of identical QVOA sequences are seen in participants $A 01, A 02$, and $A 06$, including a single dominant clone in participant A01 representing 95\% of all QVOA sequences. Rebound sequences are generally distinct from QVOA sequences, except in participant A06, in which limited genetic diversity challenged distinction and in A07, in which the single QVOA sequence aligned with a rebound lineage.

We did, however, observe exceptions to this pattern. In participant A07, who had the lowest measures of viral reservoir before the trial (IUPM, DNA), we recovered just a single virus in the pre-trial QVOA. This single QVOA viral sequence was identical to 1 of the 2 rebound virus populations (Figure 3). Participant A06 had extremely narrow viral diversity throughout the pre-ART, rebound, and QVOA sequences, making all sampled viruses, including rebound and QVOA viruses, closely related. Finally, participant A08 had 2 pre-trial QVOA sequences that were related to a minor rebound lineage (with 4 to 7 nucleotide differences between lineages).

Neutralization sensitivity to VRCO1 and autologous plasma. Many potential mechanisms exist to explain the lack of concordance between the viruses sampled by QVOA before ATI and those that reactivated in vivo during ATI, some of which were experimentally testable. First, we reasoned that the high concen- trations of VRC01 circulating in the plasma at the time of rebound may have acted as a sieve to prevent more VRC01-sensitive viruses from replicating systemically. Thus, we hypothesized that autologous neutralizing Abs circulating during ATI may have had differential pressure on certain viruses. To test this, we cloned the consensus sequence of lineages of QVOA and rebound populations, as well as selected pre-ART plasma viruses from participants A01, A02, A06, and A09 (as shown in Figures 3 and 4), and tested the Envs as pseudoviruses for neutralization sensitivity to VRC01. In each participant tested, we found similar neutralization sensitivity (by $\mathrm{IC}_{80}$ ) to VRC01 of the rebound and QVOA Envs. As shown in Figure 5, the VRC01 neutralization curves of the QVOA, rebound, and pre-ART Envs overlapped for participant A01, and the $\mathrm{IC}_{80}$ values across all 4 tested participants were not substantially different between the virus groups. 

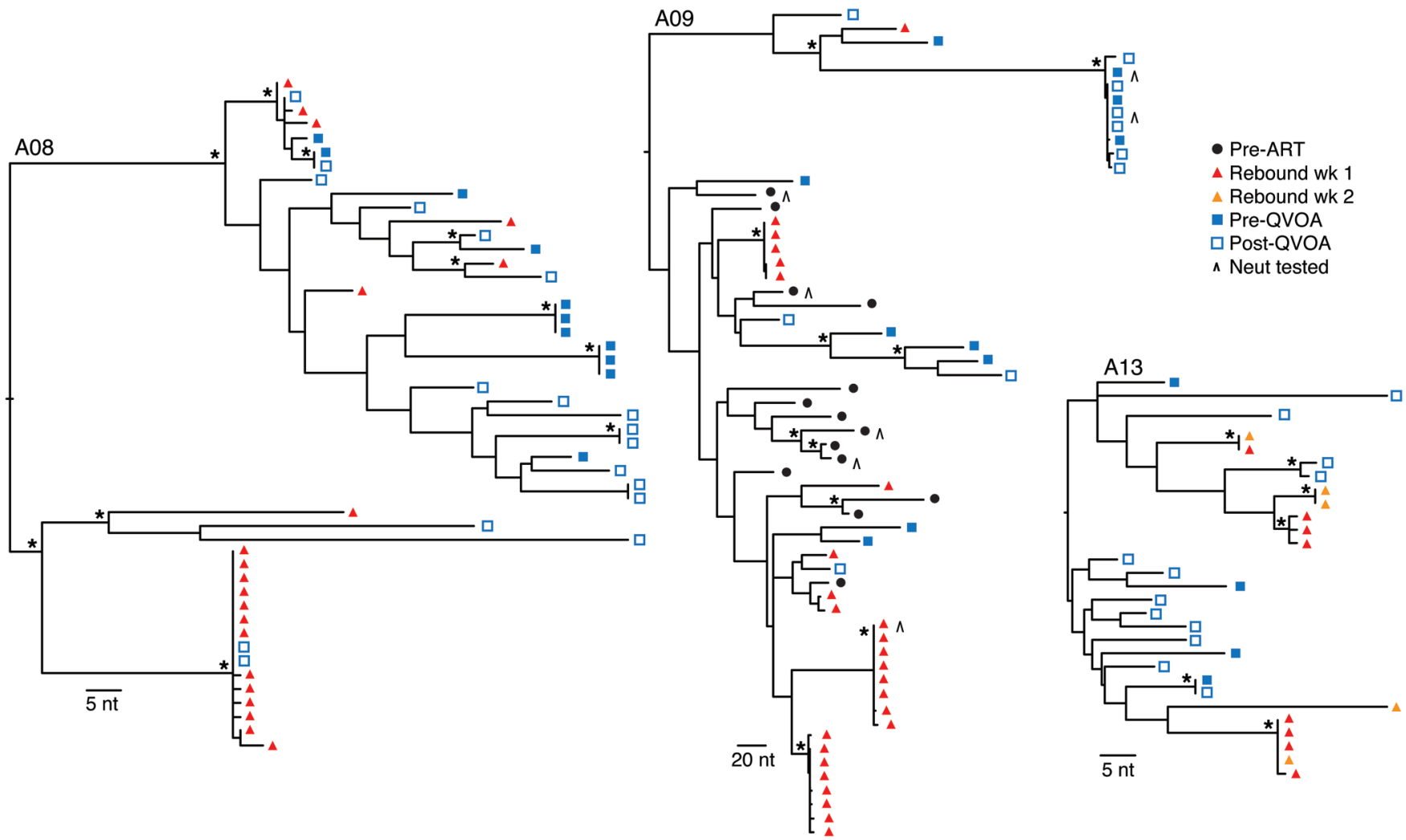

Figure 4. ML phylogenetic trees of SGS-derived gp160 env sequences from pre-ART plasma, rebound plasma, and replication-competent latent viruses for participants A08, A09, and A13. Genetic distance is shown by the scale bar, indicating 5 nucleotides (or $0.2 \%$ difference) for participants A08 and A13, and 20 nucleotides ( $0.8 \%$ difference) for participant A09. Bootstrap values above 70 are indicated by asterisks. Envs tested for neutralization sensitivity in participants $A 09$ are indicated with an inverted $v\left({ }^{\wedge}\right)$. Populations of identical QVOA sequences are seen in participants A08, A09, and A13. Viral rebound sequences are distinct from QVOA sequences in participants $A 09$ and $A 13$; in participant A08, some pre- and post-ATI QVOA sequences are identical or nearly identical to rebound lineages.

The same Env pseudoviruses were tested for sensitivity to autologous plasma collected at viral rebound, which contained both the exogenously administered VRCO1 and circulating polyclonal autologous neutralizing Abs (Figure 5). While the limited number of viruses tested in each group prevented robust statistical comparisons, we observed a trend toward greater sensitivity to plasma in QVOA viruses than in rebound viruses for all participants.

Phylogenetic analysis of intact gp160 env from resting $C D 4^{+} T$ cell proviral DNA. To examine a different surrogate of latent virus that allowed for greater sampling depth, we performed SGS of proviral DNA from the resting $\mathrm{CD}^{+} \mathrm{T}$ cells collected before and after ATI. The vast majority of integrated provirus has been shown to be defective $(21,25-27)$. To at least partially mitigate this, we restricted our analyses to sequences without evidence of mixed templates or hypermutation that encoded a complete, intact gp160 env open reading frames. We generated 546 total intact $e n v$ sequences (median of 70 per participant) and analyzed them and other sequences in within-participant ML phylogenetic trees (Figures 6 and 7).

In each participant, we found evidence for clonal expansion within the genetically intact proviral DNA compartment, with all 8 participants displaying multiple populations of identical proviral DNA sequences. Clonal populations comprised a total of $52 \%$ of intact proviral sequences at varying frequencies per participant, ranging from $5 \%$ to $78 \%$ of all proviral DNA sequences.
Two participants, A02 and A13, had expanded clones comprising more than half of the proviral DNA sequences $(61 \%$ and $78 \%$, respectively) (Figures 6 and 7). Notably, these 2 expanded proviral DNA populations were not sampled in the QVOA sequences of these participants. In general, there was detectable, but low-level, overlap between the proviral DNA and QVOA sequences across the cohort. In participant A01, for example, the clonal population representing 95\% of QVOA sequences represented only approximately $2 \%$ of the proviral DNA sequences (Figure 6). Only in participant A09 did we find the same larger expanded lineages in both QVOA and proviral DNA compartments (Figure 7).

As in the QVOA sequence analysis, the pre- and post-trial proviral DNA sequences were generally distinct from rebound viruses. The pre-trial proviral DNA sequences did not align with rebound viruses in the majority of participants (A01, A02, A05, A06, A08, and A09). Notably, the extensively expanded clonal populations in participants $\mathrm{AO} 2$ and $\mathrm{A} 13$ did not predict in vivo viral rebound. Consistent with the QVOA sequence analysis, the participant with the smallest latent reservoir, A07, served as an exception, with proviral DNA sequences aligning with rebound lineages (Figure 5). Thus, for the majority of participants, the proviral DNA sequences - even when highly enriched for a single clonal population - did not accurately predict the virus populations that would later rebound in vivo. 
A
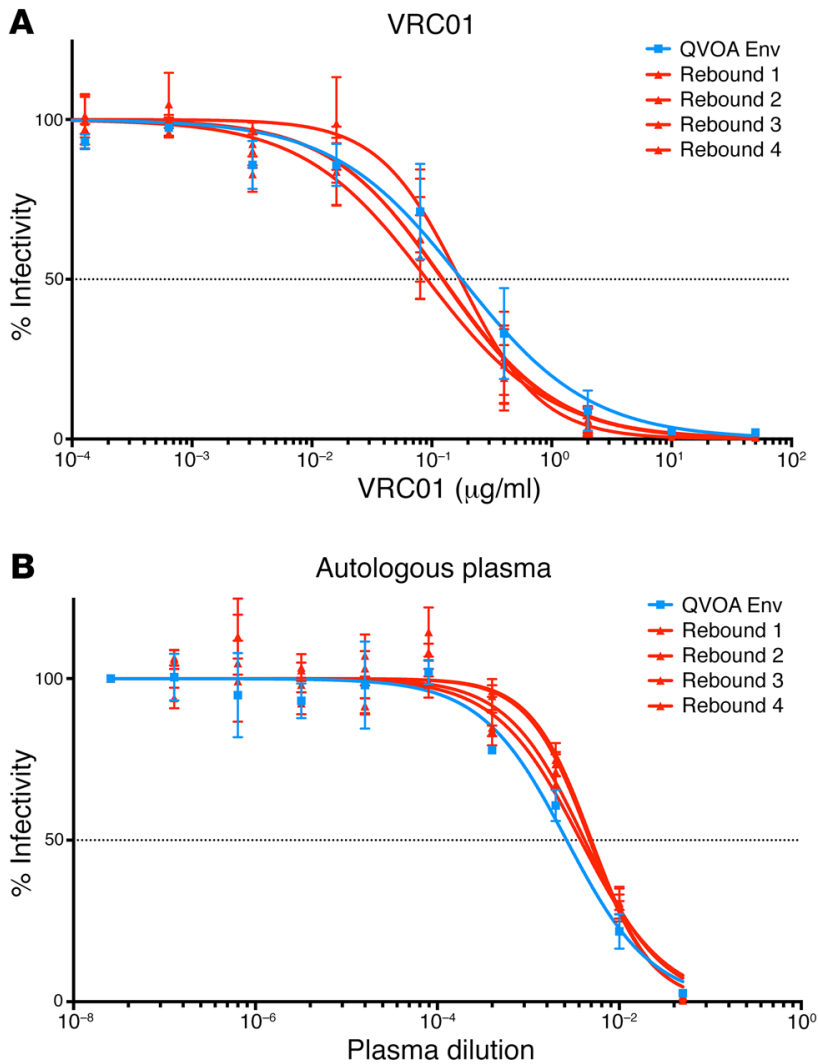

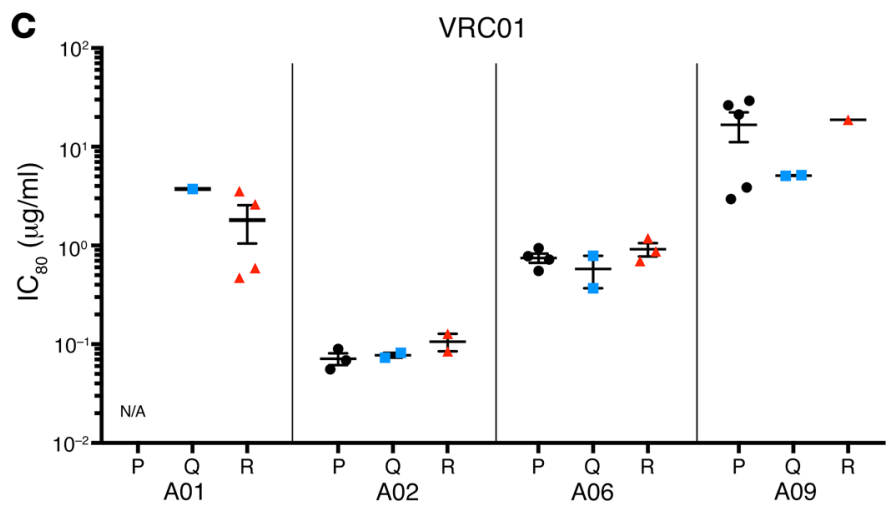

D

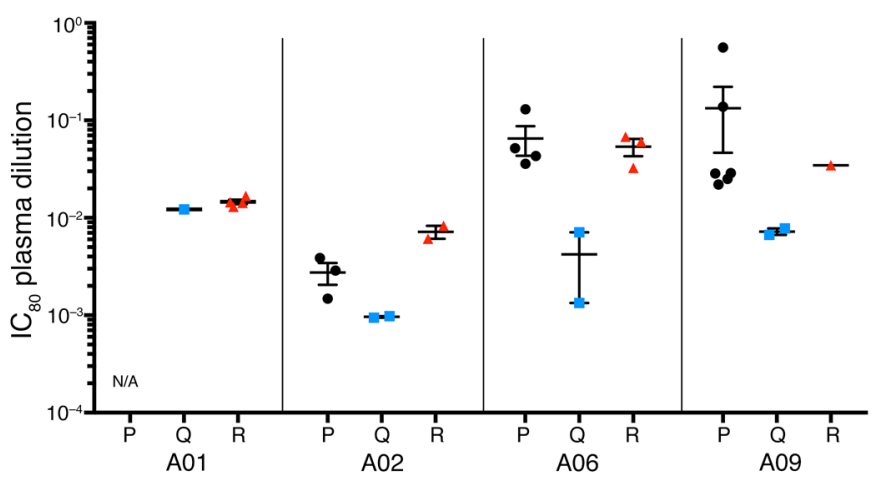

Figure 5. Neutralization sensitivity to VRCO1 and autologous plasma at the time of viral rebound. Representative Envs from 4 participants were cloned and tested as pseudoviruses in infectivity assays for sensitivity to VRCO1 and autologous plasma. Representative infectivity curves for the rebound and QVOA Envs for participant A01 are shown against VRC01 (A) and autologous plasma (B). All assays were performed in triplicate, and the mean infectivity \pm SEM for each dilution is shown. The mean $I_{80}$ for virus inhibition against VRC01 (C) and autologous plasma (D) is shown for 4 participants. Pre-ATI viruses are shown in black, QVOA viruses in blue, and rebound viruses in red.

Likewise, proviral DNA phylogenies did not indicate that viral rebound altered the post-ATI latent reservoir. In 3 participants (A01, A02, and A05), we found no post-ATI proviral DNA sequences aligned with rebound virus. Three participants had both pre- and post-ATI proviral DNA sequences that aligned with rebound at roughly the same frequency, suggesting no change due to ATI (A06, A07, and A13). In the remaining 2, we found a minor population of post-trial proviral DNA envs aligned with rebound that were not detected before the trial (5\% [1 of 21] in A08 and $6 \%$ [2 of 32] in A09). These data support the findings of QVOA sequence analysis that viral rebound did not substantially change the composition of the latent reservoir.

Statistical comparisons of virus populations. To assess the relatedness of virus compartments more objectively, we performed 2 independent analyses. To test phylogenetic relatedness, we determined genealogical sorting indices (GSI) (Table 2) (28). The GSI tests the phylogenetic similarities of 2 sequence groups, with the null hypothesis that the virus populations were the same; GSI values range from 0 (complete interspersion) to 1 (complete monophyly), with statistical significance indicating greater than random segregation between groups. Comparisons of pre- and post-ATI QVOA and proviral DNA populations indicated that the majority of participants had highly interspersed latent pre- and post-ATI virus populations. Only the QVOA sequences for participant A08 had a statistically significantly high GSI value, indicating changes in the composition of the replication-competent viruses over time. The highly interspersed pre- and post-ATI sequence sets support the concept that ATI induced no substantial changes to the sampled reservoir. Next, we grouped the pre- and post-ATI sequences together and compared QVOA and proviral DNA viruses. In 5 of 6 evaluable participants (those with $>2$ QVOA sequences per time point), we found significant $(P<0.05)$ differences between the replication-competent (QVOA) and genetically intact (proviral DNA) viruses sampled from the same resting $\mathrm{CD} 4^{+} \mathrm{T}$ cells. Finally, we compared QVOA and proviral DNA viruses with rebound viruses. In all evaluable participants, the latent viral sequences differed significantly $(P<0.001)$ from those of the rebound virus populations.

Next, we used a non-tree-based method examining sequence differences to compare the same virus populations. For each participant, we calculated the pairwise Levenshtein edit distance (LD) between all sequences. We then performed pairwise comparisons between virus populations using pairwise permutational multivariate analysis of variance (PERMANOVA) to test the null hypothesis that the distributions of LDs were the same when comparing virus populations with themselves and with other virus populations (Supplemental Figure 4). As with GSI, we first compared pre- and post-ATI QVOA and proviral DNA sequences and found that they were highly similar (Supplemental Figure 4, left 

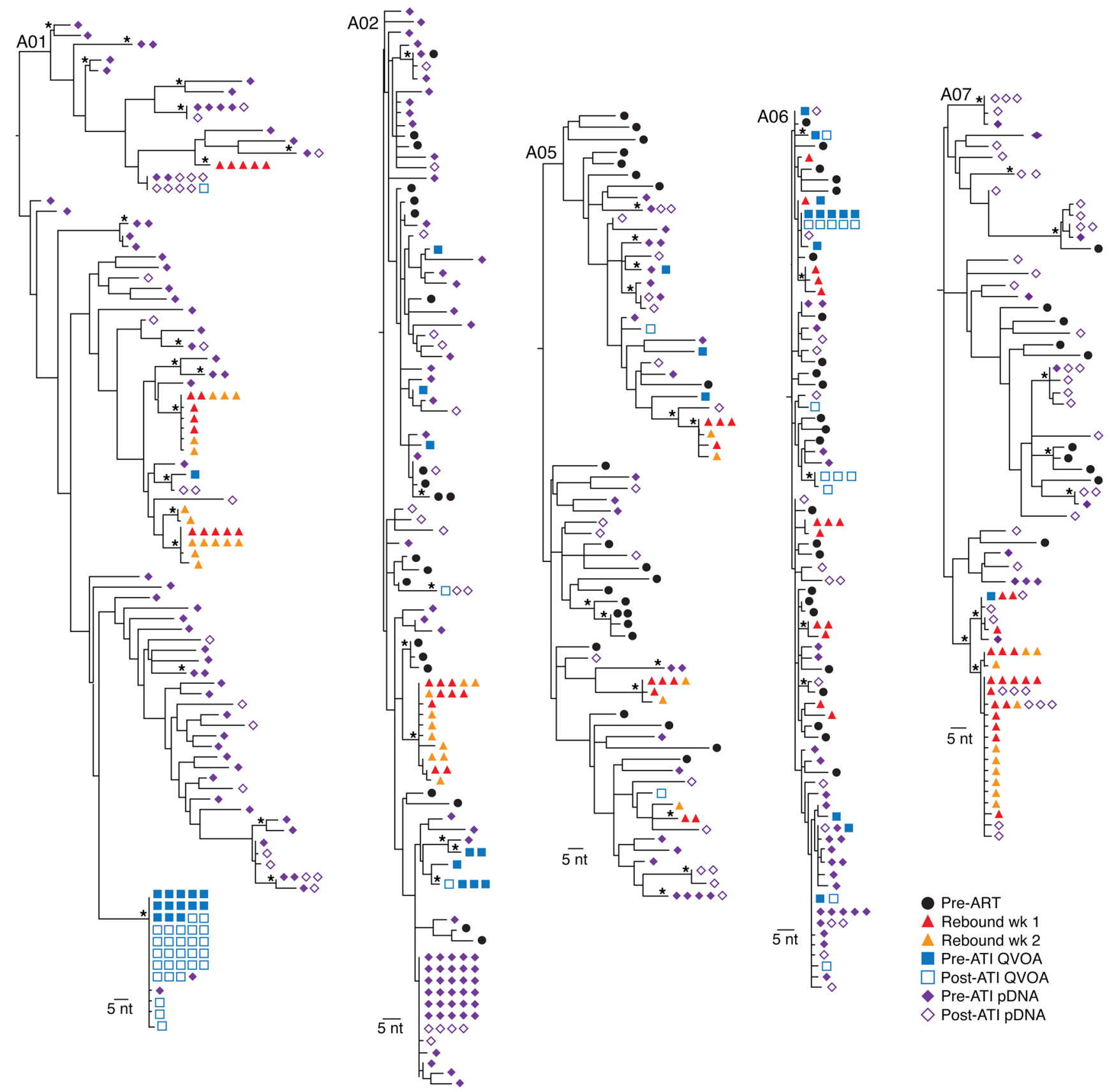

Figure 6. ML phylogenetic trees of SGS-derived gp160 env sequences from pre-ART plasma, rebound plasma, replication-competent latent viruses, and genetically intact proviral DNA for participants A01, A02, and A05-A07. Env sequences from pre-ART plasma are shown as black circles, sequences from rebound plasma from the first and second weeks of detectable viremia after ATI are shown as red and orange triangles, respectively, sequences from virus supernatant from pre- and post-ATI individual p24+ QVOA culture well supernatants are shown as solid blue or open blue squares, and sequences from pre- and post-ATI proviral DNA (pDNA) are shown as solid purple or open purple diamonds. For clearer visualization, identical sequences are aligned horizontally in rows of 5 . Genetic distance is shown by the scale bar, indicating 5 nucleotides (or $\sim 0.2 \%$ difference) in all trees. Bootstrap values above 70 are indicated by asterisks. Populations of identical proviral DNA sequences are seen in all participants, including a large clonal population in participant A02 comprising $61 \%$ of total proviral DNA sequences. In general, the larger clonal populations of proviral DNA sequences show limited or no overlap with the larger QVOA clones.

box for each participant). Then, we grouped the pre- and postATI sequences together and compared proviral DNA with QVOA sequences (Supplemental Figure 4, right box for each participant). Here, we found statistical significance in the same participants as with the GSI method, with the exception of participant A09, in whom an extremely high intrapopulation genetic diversity pre- vented distinction between QVOA and proviral DNA sequences. Finally, comparisons of rebound sequences with QVOA and proviral DNA showed highly significant differences in all participants except those with limited or narrow QVOA sequence populations (A06 and A07). Thus, both statistical methods supported phylogenetic analysis results suggesting that ATI did not substantially 

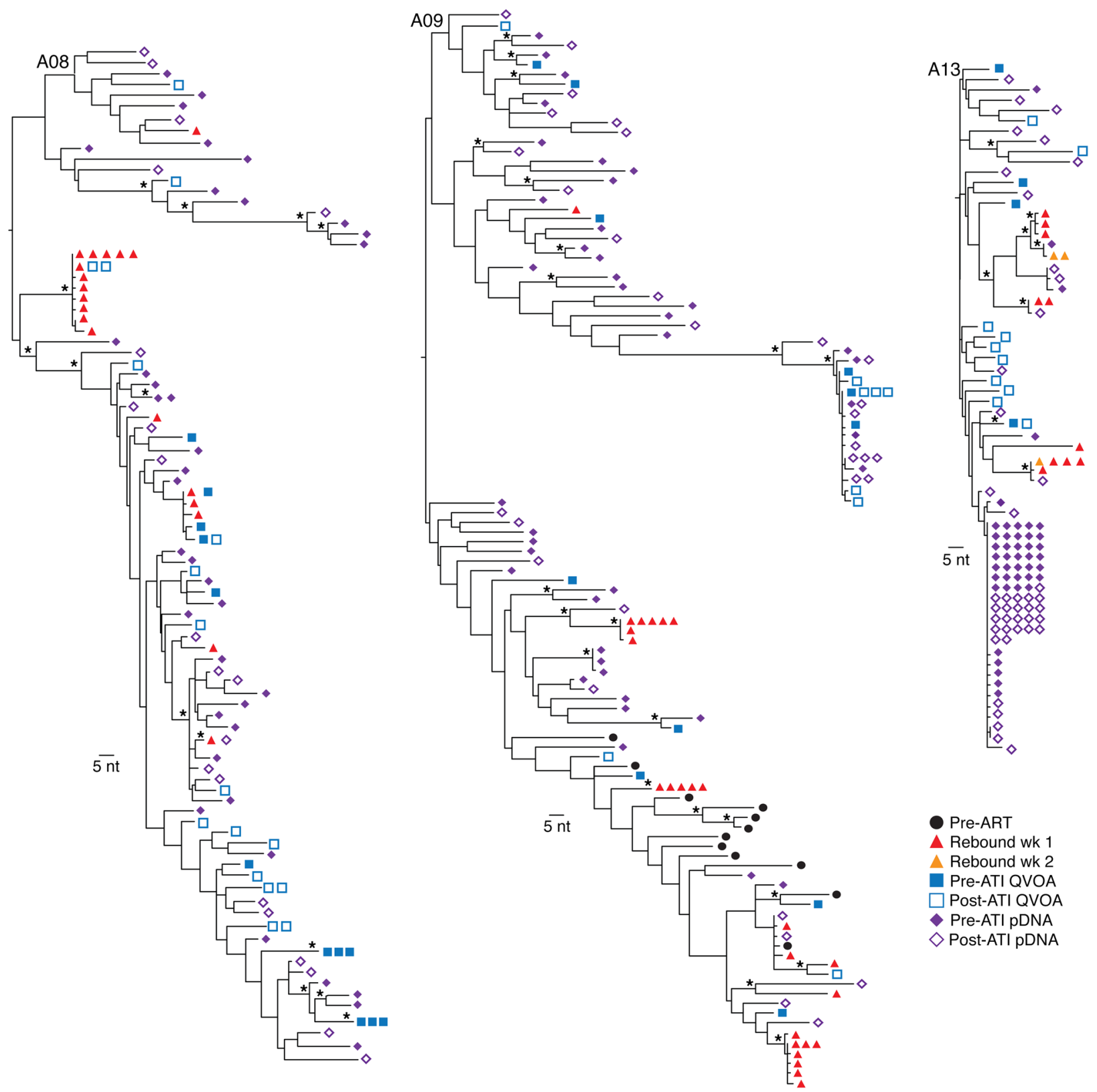

- Pre-ART

Rebound wk 1

Rebound wk 2

Pre-ATI QVOA

$\square$ Post-ATI QVOA

- Pre-ATI pDNA

$\diamond$ Post-ATI pDNA

Figure 7. ML phylogenetic trees of SGS-derived gp160 env sequences from pre-ART plasma, rebound plasma, replication-competent latent viruses, and genetically intact proviral DNA for participants A08, A09, and A13. Populations of identical proviral DNA sequences are found in all participants, including a single clone comprising $78 \%$ of the proviral DNA sequences for participant A13, which was not sampled in the QVOA sequences. Bootstrap values above 70 are indicated by asterisks.

change the composition of the reservoir and that the QVOA, proviral DNA, and rebound virus populations all differed substantially from each other.

\section{Discussion}

Clinical trials of novel HIV therapy and cure strategies in HIVinfected individuals commonly use ATIs $(13,17,29,30)$. Concerns remain, however, regarding their safety and effects on reseeding the stable latent reservoir. Here, we used the extensive sampling available in the clinical trial A5340 to assess the effect of ATIs in HIV-infected individuals who initiated ART during chronic infection. We assessed peripheral latent virus after at least 6 months of suppressive ART, allowing us to measure the stable latent reservoir $(31,32)$. We found no significant change in the size or composition of the peripheral reservoir. Quantitative measures of viral transcripts (total HIV-1 DNA and cell-associated RNA) in CD4 ${ }^{+} \mathrm{T}$ cells showed no statistical increase. Although PCR-based assays of latent virus measure predominantly defective virus $(26,27)$, the lack of increase of any integrated virus after ATI by these measures is reassuring. The measures of resting $\mathrm{CD} 4^{+} \mathrm{T}$ cells harboring replication-competent virus also showed no significant differences between pre- and post-ATI samples. Further, the correlation between the measures of replication-competent virus and total HIV-1 DNA (Supplemental Figure 1) suggests that we measured 
Table 2. Geneological Sorting Indices

\begin{tabular}{|c|c|c|c|c|c|c|c|c|c|c|}
\hline \multirow[b]{2}{*}{ Participant } & \multicolumn{2}{|c|}{ QVOA pre- and post-ATI } & \multicolumn{2}{|c|}{ Proviral DNA pre- and post-ATI } & \multicolumn{2}{|c|}{ QVOA vs. proviral DNA } & \multicolumn{2}{|c|}{ QVOA vs. rebound } & \multicolumn{2}{|c|}{ Proviral DNA vs. rebound } \\
\hline & Before ATI & After ATI & Before ATI & After ATI & QVOA & Proviral DNA & QVOA & Rebound & Proviral DNA & Rebound \\
\hline A01 & 0 & 0.061 & 0.029 & 0.13 & $0.42^{\mathrm{A}}$ & 0 & $0.89^{A}$ & $0.84^{A}$ & $0.87^{\mathrm{A}}$ & $0.56^{\mathrm{A}}$ \\
\hline A02 & 0 & 0.012 & $0.19^{B}$ & $0.2^{A}$ & $0.2^{\mathrm{A}}$ & $0.43^{A}$ & $0.86^{A}$ & $1.0^{A}$ & 0.94 & $1.0^{A}$ \\
\hline A05 & 0.33 & 0 & 0.13 & 0.10 & 0.10 & 0 & $0.15^{\mathrm{B}}$ & $0.46^{B}$ & $0.67^{A}$ & $0.30^{A}$ \\
\hline A06 & 0.166 & 0.05 & 0.048 & $0.10^{B}$ & $0.10^{\mathrm{B}}$ & $0.12^{\mathrm{B}}$ & $0.54^{A}$ & $0.53^{A}$ & $0.49^{A}$ & $0.50^{A}$ \\
\hline A07 & NA & NA & 0.06 & NA & NA & NA & NA & NA & $0.12^{\mathrm{B}}$ & $0.55^{A}$ \\
\hline A08 & 0.19 & $0.34^{\mathrm{B}}$ & 0.063 & 0.16 & 0.16 & $0.29^{A}$ & 0.032 & $0.38^{A}$ & $0.62^{A}$ & $0.16^{B}$ \\
\hline A09 & 0.05 & 0 & 0.06 & 0.11 & 0.11 & 0.079 & $0.53^{A}$ & $0.50^{A}$ & $0.61^{A}$ & $0.4^{\mathrm{A}}$ \\
\hline $\mathrm{A} 13$ & 0.13 & 0 & $0.088^{B}$ & $0.39^{A}$ & $0.39^{A}$ & $0.11^{\mathrm{B}}$ & $0.64^{A}$ & $0.16^{B}$ & $0.21^{A}$ & $0.48^{A}$ \\
\hline
\end{tabular}

${ }^{\mathrm{A}} P<0.001 ;{ }^{\mathrm{B}} P \geq 0.001$ to $P \leq 0.05$.

biologically meaningful values and that they did not change consistently across this cohort with ATI.

In parallel with quantitative measures, phylogenetic analyses showed no substantial difference in the composition of the peripheral latent reservoir before or after ATI. We compared preand post-ATI QVOA viral sequences and found minimal changes among the sampled replication-competent virus populations, with no evidence that viruses replicating during rebound significantly enriched the replication-competent peripheral latent reservoir after ATI. Likewise, we found that genetically intact proviral DNA virus populations sampled after the trial did not align more closely with rebound viruses in the majority of the participants. In the 2 exceptions, rebound-like viruses represented only a small proportion of total post-trial viruses $(5 \%-6 \%)$.

Indeed, in most participants, the phylogenetic composition of virus populations induced from and directly sequenced from resting $\mathrm{CD} 4^{+} \mathrm{T}$ cells were remarkably stable over the approximately 9 to 12 months between samplings, despite ATI. Recent reports have described a waxing and waning of clonally expanded virus populations over time (33), but we found many identical sequences in the QVOA and proviral DNA sequences between the 2 time points and similar frequencies of expanded clonal populations over this relatively short time period (Figures 6 and 7). Phylogenetic and non-tree-based statistical tests supported this conclusion, showing no statistical differences between the sampled latent viruses over time (Table 2 and Supplemental Figure 4). Importantly, this finding held true for participants with very small or narrow reservoirs, which is relevant for trials proposing ATIs for individuals with earlier ART initiation whose reservoirs may be smaller and less diverse (34-36). Participant A07, for example, had very low measures of HIV-1 DNA and cell-associated RNA, as well as similarly low pre- and post-trial IUPM quantifications, with no inducible virus detected in the post-ATI QVOA (Figure 2). Remarkably, sequencing of the single induced virus in the pre-trial QVOA revealed a gp160 env that was identical to a rebound lineage. Together, these results provide reassurance that, in addition to being clinically safe, short ATIs with rapid reinitiation of ART that are conducted within the context of a clinical trial do not substantially increase the size or diversity of the latent reservoir. As the field moves toward more permissive treatment interruptions of longer duration, tolerating higher levels of viremia prior to ART reinitiation (37), these types of analyses should be reconsidered.

A salient finding of this study is the extent of clonal expansion within the replication-competent and genetically intact viruses comprising the peripheral latent reservoir. Clonally expanded cells harboring provirus have long been recognized as an important component of latently infected cell populations (1-3) and the substrate of low-level viremia in individuals on ART $(38,39)$. While clonal populations encode predominantly defective virus $(21,25-27)$, recent reports identifying identical genetically intact and/or replication-competent viruses $(20-22,40)$ illustrate the importance of clonally expanded populations in the persistent latent reservoir that, crucially, can reactivate to reestablish systemic viral replication. Clonal expansion of latently infected cells can only be definitively proven through identification of identical whole proviral genomes in multiple cells with the same integration site, but these methods are highly resource intensive. The clonal sequence index (silicianolab.johnshopkins.edu/cps) indicates that identical env sequences generated with the primers used in our study are $95 \%$ likely to predict identical whole-genome sequences (19). Further, recent mathematical and experimental evidence suggests that identical sequences are highly likely to predict clonally expanded latent cell populations (19-21). Studies using sequence analysis of replication-competent or intact sequences demonstrate high frequencies of expanded clonal populations: Lorenzi et al. found that $54 \%$ of the sequences were clonal in both the viral outgrowth and proviral DNA populations in 4 ARTsuppressed individuals (40); Hosmane et al. found that $57 \%$ of the sequences were identical in the viral outgrowth sequences of 12 individuals (22); and Lee et al. found that $62 \%$ of the intact proviral DNA genomes were identical in 3 individuals (21). Here, we found a similarly high prevalence of clonal populations in the QVOA and proviral DNA sequences.

We found expanded clonal populations in all participants with sufficient QVOA sequences to evaluate and within all participants' genetically intact proviral DNA. In the 4 participants with ample QVOA sequences, we found that $68 \%$ of the QVOA sequences fell within a clonal population and that several participants had highly expanded clones representing a majority of the sampled replication-competent virus. The QVOA sequences of participant A01 contained a single clone representing $95 \%$ of the 
sampled virus, and $55 \%$ of the QVOA sequences for participant A02 fell into 2 clonal lineages. We found similarly high levels of clonal viruses in genetically intact proviral DNA sequences, with clonal sequences in all participants at a total frequency of $52 \%$ and single, expanded clones comprising more than half of all proviral DNA sequences in 2 participants.

As reported by Lorenzi et al., we also observed noticeably different distributions of these expanded clones in the QVOA and intact genes of individual participants. The GSI values and pairwise LD analyses further indicate that these methods sample distinct virus populations. The standard QVOA assay is known to underrepresent the full range of latently infected cells present in cells used in the assay, as some infected cells are not inducible with a single stimulation $(22,26)$. Genetically intact proviral sequences may overrepresent the reservoir, because viruses with markedly decreased fitness or fatal defects may appear grossly normal by sequence analysis. In our study, 2 independent statistical methods revealed that the frequency of expanded clonal populations was largely consistent between samples over the 6-12 months between sampling, but differed significantly between QVOA and proviral DNA sequence compartments at both time points.

The unique sampling available in A5340 allowed us to link these reservoir sequences to the viruses that reactivated spontaneously upon ATI in each participant. Notably, we found a striking discordance between the latent sequences (both QVOA and intact proviral DNA) and rebound viruses. Inadequate depth of sampling could explain much of this discordance. The viruses that rebound during ATI represent only a small fraction of a potentially large and diverse latent reservoir capable of reactivating over time. Likewise, viruses that reactivate from approximately 30.5 million resting $\mathrm{CD}^{+} \mathrm{T}$ cells after ex vivo stimulation in a QVOA assay represent a small fraction of reservoir viruses. Sampling the same peripheral resting memory $\mathrm{T}$ cells via direct sequencing of viral DNA expanded the number of sequences, but was still limited to latently infected cells circulating in the periphery at the time of sampling. Yet, when the sampled reservoir viruses are composed overwhelmingly or primarily of one or a few expanded clones (e.g., 95\% QVOA sequences in participant $\mathrm{A} 01$ or $55 \%$ in participant $\mathrm{A02}$ ), that clonal population should be represented in the rebound virus if the 2 samples are drawing from the same pool of replication-competent viruses in an unbiased manner. Only in the participants with very small or narrow latent reservoirs (A06, A07) did the presence of a dominant clonally expanded population in pre-trial sampling predict the virus that would contribute to viral reactivation upon ATI. The lack of correlation between rebound viruses and our best attempts at sampling the latent reservoir suggests that our sampling methods and in vivo reactivation are governed by different principles. The practical implication of the discordance between the replication-competent virus populations sampled peripherally and those that reactivated in vivo is that efforts at pre-trial sampling for phenotypic screening, for example, will be highly challenging.

There are several potential explanations for why pre-ATI reservoir sampling did not predict viral rebound, some of which we were able to test experimentally. We found similar VRC01 sensitivities between the QVOA viruses and rebound viruses, suggesting that a VRC01 "sieve effect" did not prevent latent viruses from rebounding. Autologous plasma, in contrast, did differentiate (nonsig- nificantly) the virus populations in 2 participants, suggesting that autologous Abs may drive the selection of rebound viruses. Additional explanations for the lack of QVOA and rebound virus concordance that we were not able to test, but that merit future study, include anatomic compartmentalization of reactivating viruses, differences between ex vivo stimulation in QVOA and in vivo reactivation, including antigen-specific stimulation, quiescent $\mathrm{T}$ cell subsets resistant to ex vivo stimulation, and a host of other epigenetic and integration site-related controls of virus reactivation.

Our study has several important limitations. First, it is a small study with only 9 male participants and is limited to peripheral sampling. Future work in larger, more diverse cohorts with more extensive sampling will be important. We studied a limited ATI that resulted in a short period of viremia in adults who initiated ART during chronic infection. We caution that the results should not be extrapolated to distinct groups, including individuals who initiate ART during acute infection or those who undergo longer ATIs. Our clinical trial also included an intervention, i.e., infusion of the bnAb VRC01, which may have had unmeasured effects on the reservoir. We tested the role of a neutralization sieve effect and found no difference between rebound and QVOA viruses. Further, we note that viral rebound in the majority of trial participants occurred with the same kinetics and clonality as observed in historical controls, arguing for no additional effects of VRCO1 on rebound viremia (17). Other effects, however, including Abdependent cellular virus inhibition or $\mathrm{Ab}$-induced immunomodulation have not been thoroughly tested.

In summary, our study demonstrated that ATIs with transient viremia did not substantially alter the size or diversity of the peripheral reservoir in this cohort. These results build on the findings of a recent clinical trial involving ATI conducted by Clarridge et al., who found no change in quantitative measures of the preand post-ATI reservoir (41). Together, the results provide reassurance that short ATIs can be safely performed in the evaluation of therapeutic and curative strategies for HIV-infected patients. Our study also emphasizes the prevalence of clonally expanded virus populations in the latent reservoir, highlights the substantial variability in the frequency of these clones depending on the sampling method, and shows that the frequency of such clones before ATI does not predict their contribution to viral rebound.

\section{Methods}

Study participants. The ACTG clinical trial A5340 (ClinicalTrials.gov NCT02463227) tested the safety and ability of the bnAb VRC01 to maintain viral suppression during ATI. The study design, approvals, and results were published previously (17). All A5340 trial participants who opted to undergo leukapharesis were included in this study $(n=$ 9). Participant A14 was excluded from the qualitative analysis because of an insufficient number of rebound plasma env sequences and a limited number of $\mathrm{p} 24^{+}$QVOA wells.

Quantification of total HIV-1 DNA and cell-associated RNA. CD4 ${ }^{+} \mathrm{T}$ cells were isolated from PBMCs using an EasySep Human $\mathrm{CD}^{+} \mathrm{T}$ cell Enrichment Kit (STEMCELL Technologies). Cellular RNA and DNA from isolated $\mathrm{CD} 4{ }^{+} \mathrm{T}$ cells were purified using the AllPrep DNA/RNA Kit (QIAGEN) as specified by the manufacturer, quantified using a NanoDrop ND-1000 spectrophotometer (Thermo Fisher Scientific), and normalized to cell equivalents by qPCR using human genomic TERT 
for DNA and RPLPO expression for RNA (Life Technologies, Thermo Fisher Scientific). Total cellular HIV-1 DNA and RNA were quantified with a qPCR TaqMan assay using the LTR-specific primers F522-43 (5'-GCCTCAATAAAGCTTGCCTTGA-3'; HXB2522-543) and R62643 (5'-GGGCGCCACTGCTAGAGA-3'; 626-643), coupled with a FAMBQ probe (5'-CCAGAGTCACACAACAGACGGGCACA-3') using the QuantStudio 6 Flex Real-Time PCR System (Applied Biosystems). The cell-associated HIV-1 DNA copy number was determined using a reaction volume of $20 \mu \mathrm{l}$ with $10 \mu \mathrm{l}$ of $2 \times$ TaqMan Universal Master Mix II with UNG (Life Technologies, Thermo Fisher Scientific), 4 pmol of each primer, 4 pmol probe, and $5 \mu \mathrm{l} \mathrm{DNA}$. Cycling conditions were $50^{\circ} \mathrm{C}$ for 2 minutes, $95^{\circ} \mathrm{C}$ for 10 minutes, and then 60 cycles of $95^{\circ} \mathrm{C}$ for 15 seconds and $59^{\circ} \mathrm{C}$ for 1 minute. The cell-associated HIV-1 RNA copy number was determined in a reaction volume of $20 \mu \mathrm{l}$ with $10 \mu \mathrm{l}$ of a $2 \times$ TaqMan RNA-to-Ct 1-Step Kit (Life Technologies, Thermo Fisher Scientific), $4 \mathrm{pmol}$ of each primer, $4 \mathrm{pmol}$ probe, $0.5 \mu \mathrm{l}$ reverse transcriptase, and $5 \mu \mathrm{l}$ RNA. Cycling conditions were $48^{\circ} \mathrm{C}$ for 20 minutes, $95^{\circ} \mathrm{C}$ for 10 minutes, and then 60 cycles of $95^{\circ} \mathrm{C}$ for 15 seconds and $59^{\circ} \mathrm{C}$ for $1 \mathrm{~min}$ ute. For HIV-1 DNA measurements, external quantitation standards were prepared from the ACH-2 cell line in a background of HIV-1-negative human cellular DNA and calibrated to the Virology Quality Assurance (VQA) (NIH DAIDS) cellular DNA quantitation standards. For HIV RNA measurements, external quantitation standards were prepared from full-length NL4-3 RNA, followed by copy number determination using the Abbott RealTime assay (Abbott Diagnostics) and calibrated to VQA HIV-1 RNA standards. Patient specimens were assayed with up to 800 ng total cellular RNA or DNA in replicate reaction wells and the copy number determined by extrapolation against a 7-point standard curve (1-10,000 cps) performed in triplicate. DNA values for 1 participant measured under the limit of detection with fewer than 21.4 copies/ million $\mathrm{CD}^{+} \mathrm{T}$ cells were censored at 21.4 copies/million $\mathrm{CD} 4^{+} \mathrm{T}$ cells throughout the analyses. DNA values for A06 are not shown because of assay failure secondary to a primer mismatch.

Single-copy assay. Residual plasma HIV viremia was measured using the validated ultrasensitive integrase single-copy assay (iSCA) (42). Plasma from the participants was spiked with an internal RCAS virion (43) standard, and qPCR reactions were performed with a Roche LightCycler 480 system using primers and probes specific to a conserved area of the HIV integrase gene.

QVOA. Resting CD4 ${ }^{+} \mathrm{T}$ cells (30.5 million) from leukapheresisderived PBMCs from individual HIV-1-infected participants and purified by 2 -step magnetic bead depletion $\left(\mathrm{CD} 4^{+} \mathrm{T}\right.$ cell Isolation Kit and CD69 Microbead Kit II, both from Miltenyi Biotec). Resting CD4 ${ }^{+} \mathrm{T}$ cells were then serially diluted 5 -fold, briefly cocultured with a 10-fold excess of $\gamma$-irradiated allogeneic PBMCs from uninfected donors, and stimulated with RPMI containing phytohemagglutinin (PHA) (Remel), 10\% FBS $100 \mathrm{U} / \mathrm{ml} \mathrm{IL-2} \mathrm{(Novartis),} \mathrm{and} \mathrm{1 \%} \mathrm{T} \mathrm{cell} \mathrm{growth}$ factor (produced by the Siliciano laboratory as previously described, ref. 44). Media were removed 1 day after mitogen stimulation, and target MOLT-4/CCR5 cells were added to fresh media to propagate replication-competent HIV-1. The ratios of target cells were $1 \times 10^{7}$ to $1 \times 10^{6}$ patient resting CD $4^{+} \mathrm{T}$ cells to $2.5 \times 10^{6}$ target cells. Five days after stimulation, culture media were replaced, and cells in each well were split. Supernatants from each well were then assessed for p24 protein by qPCR and ELISA to determine the presence of replicationcompetent virus on days 14 and 21 (Alliance HIV-1 p24 antigen ELISA Kit, PerkinElmer). The frequency of latently infected cells among the input resting $\mathrm{CD} 4{ }^{+} \mathrm{T}$ cells was calculated by a ML method, as described previously, and expressed as IUPM, and 95\% CIs for individual IUPM determinations were $\pm 0.7 \log$ IUPM, or 5 -fold (44). Using the ML method, samples without replication-competent virus were assessed in analyses as the limit of detection of the assay, based on cell input. Supernatant from wells containing replication-competent virus was then collected and processed by SGS methods.

$S G S$ of replication-competent virus from QVOA wells. Viral RNA from p24 ${ }^{+}$wells collected in MOLT-4/CCR5 QVOA assays was extracted using the QIAmp viral RNA Mini Kit (QIAGEN) according to the manufacturer's instructions. HIV cDNA was generated by incubating $30 \mu$ l template RNA with $3 \mu \mathrm{l} 10 \mathrm{mM}$ dNTPs and $0.75 \mu \mathrm{l}$ of $20 \mu \mathrm{M}$ antisense primer R3B3R (5'-ACTACTTGAAGCACTCAAGGCAAGCTTTATTG-3') for 5 minutes at $65^{\circ} \mathrm{C}$ to denature the RNA, followed by reverse transcription using SuperScript III Reverse Transcriptase (Life Technologies, Thermo Fisher Scientific) according to the manufacturer's instructions. Amplification of gp160 env genes was performed by nested PCR using Platinum Taq High Fidelity Polymerase (Life Technologies, Thermo Fisher Scientific) as previously described (17). In order to ensure that a majority of sequences would be generated from a single template, cDNA was diluted to yield less than $30 \%$ PCR-positive products on a precast $1 \%$ agarose gel (Thermo Fischer Scientific). Three to eight amplicons per QVOA sample were sequenced on an Illumina MiSeq platform; only wells with identical consensus sequences (85\% identity at each nucleotide position) were used for analysis. Divergent sequences derived from the same QVOA well indicate evidence of multiple viruses, and were excluded from analysis. Sequences that contained stop codons, large deletions, or mixed bases were also excluded from further analysis. Sequences were codon aligned using MUSCLE in the Geneious suite (version 8.1.7), manually refined, and gap-stripped. Phylogenetic trees were inferred using ML/rapid bootstrapping in RAxML, version 8, run on the Cyberinfrastructure for Phylogenetic Research (CIPRES) Science Gateway (http://www.phylo.org/). Evolutionary models were chosen for each participant data set using jModelTest (version 2.1.4), and model parameters were estimated concurrently with the phylogram topology. Trees were mid-point rooted and visualized using MEGA, version 621, and bootstraps greater than 70 are shown in the figures. All sequences were deposited in GenBank (accession numbers MH263749 and MH264427).

SGS of proviral DNA from pre- and post-trial resting CD4 $4^{+} \mathrm{T}$ cells. Viral DNA was extracted from multiples of 4 million resting $C D 4^{+} \mathrm{T}$ cells from pre- and post-trial time points according to the manufacturer's instructions (QIAamp DNA Mini and Blood Mini Kit, QIAGEN). Amplification of gp160 env genes was performed as described above. Amplicons were run on precast $1 \%$ agarose gels (Thermo Fisher Scientific), and bands of appropriate size were directly sequenced on the Illumina MiSeq platform. Sequences were inspected for evidence of priming from multiple templates, stop codons, large deletions, or introduction of PCR error in early cycles; a threshold of $85 \%$ identity at each nucleotide position was used. Only sequences with full gp160 env ORFs and no evidence of multiple templates were used in analysis. Sequences were codon aligned using MUSCLE in the Geneious suite (version 8.1.7), manually refined, and gap-stripped.

Env cloning, pseudovirus titering, and neutralization assays. Select env sequences from pre-ART, rebound, and pre-trial QVOA samples were molecularly cloned for the production of pseudovirus and phenotypic analyses as described previously (17). Amplicons identified 
by gel electrophoresis were gel purified using the QIAquick Gel Purification Kit (QIAGEN), ligated into the pcDNA $3.1 \mathrm{Zeo}^{(+)}$Expression Vector (Invitrogen, Thermo Fisher Scientific), and transformed into NEB 5-alpha Competent E. coli (New England BioLabs). Transformed bacteria $(100 \mu \mathrm{l})$ were plated onto Luria broth (LB) agar plates supplemented with $50 \mu \mathrm{g} / \mathrm{ml}$ ampicillin and cultured at $37^{\circ} \mathrm{C}$ for 24 hours. Single colonies were selected and grown overnight in liquid LB broth at $37^{\circ} \mathrm{C}$ with shaking at $250 \mathrm{rpm}$, followed by plasmid isolation. Finally, each molecular clone was sequence confirmed against the reference env amplicon using the Illumina MiSeq platform. Pseudovirus was prepared as described previously (17). Culture supernatants were harvested 48 hours after transfection, and pseudoviruses were titrated on TZM-bl reporter cells. Virus neutralization by VRC01 or heat-inactivated autologous plasma collected at the first detectable rebound was assessed on TZM-bl cells as described previously (17). All assays were performed in triplicate in each of at least 2 independent experiments. To calculate the concentration of VRC 01 or dilution of autologous plasma that neutralized $50 \%\left(\mathrm{IC}_{50}\right)$ and $80 \%\left(\mathrm{IC}_{80}\right)$ of the viral infection, the $\mathrm{Ab}$ dose-response curves were fitted with a 4-parameter logistic regression equation using GraphPad Prism 5.0 (GraphPad Software). The bnAb VRC01 was obtained from the NIH AIDS Reagent program.

Statistics. Given the small sample size $(n=9)$, nonparametric tests were used for all comparisons. The test for within-person changes from pre-ATI to post-ATI in measures of the latent reservoir was performed using a Wilcoxon signed-rank test. Spearman's correlation testing was used to test the association between measures. Analyses were conducted in SAS, version 9.4 (SAS Institute). A $P$ value of less than 0.05 was considered statistically significant.

GSI. To assess similarities between sampled virus populations, within-person ML phylogenies of combinations of QVOA sequences, proviral DNA sequences, and viral rebound sequences were inferred using PhyML version 3.0, and GSI values were calculated using the genealogical Sorting $\mathrm{R}$ package (http://molecularevolution.org/ software/phylogenetics/gsi/download) (28). Statistical significance was assessed by randomly permuting character states across the tips of the tree 10,000 times. Nonsignificant values indicate the absence of compartmentalization ( $P$ values were corrected for multiple tests).
Pairwise PERMANOVA of LDs. For each participant, we calculated the pairwise PERMANOVA of the LD between nucleotide sequence sets that were aligned and gap-stripped. We then performed pairwise comparisons between all sample populations using PERMANOVA to test the null hypothesis that the centroids and spreads of the 2 populations were the same. $P$ values were adjusted by Bonferroni's correction for the number of tests $[n \times(n-1) / 2]$.

Study approval. The ACTG clinical trial A5340 was conducted under approval of the IRBs of the University of Pennsylvania and the University of Alabama at Birmingham. Written informed consent was obtained from all participants. The study protocols and results were published previously (17).

\section{Author contributions}

RT, RK, JH, ETO, PT, and KJB designed and conducted the A5340 clinical trial. DBS, FM, TS, SB, JL, JL, MAM, FB, RFS, JMS, and KJB conducted experiments. DBS, YZ, FM, MAM, SSM, KM, FB, RFS, JMS, PT, and KJB analyzed data. DBS, YZ, FM, MAM, SSM, ETO, FB, RFS, JMS, PT, and KJB wrote the manuscript.

\section{Acknowledgments}

We are grateful for the study volunteers of the ACTG A5340 clinical trial, who participated in the present study. The A5340 clinical trial was supported by the NIAID (U01AI068636); the Penn Center for AIDS Research (P30 AI045008); the Penn Clinical Trials Unit (AI069534); the UAB Center for AIDS Research (P30 AI027767), the UAB Clinical Trials Unit (AI069452); and the ACTG Statistical and Data Analysis Center (UM1 AI068634). A5340 viral analyses were supported by the NIAID (1-R21-AI118431); the Penn Center for AIDS Research Viral and Molecular Core (P30 AI045008); the BEAT-HIV: Delaney Collaboratory to Cure HIV-1 Infection by Combination Immunotherapy (UM1AI26620); and CARE: Delaney Collaboratory for AIDS Eradication (UM1AI126619).

Address correspondence to: Katharine J. Bar, 502D Johnson Pavilion, 3610 Hamilton Walk, Philadelphia, Pennsylvania 19104, USA. Phone: 215.573.8497; Email: bark@pennmedicine.upenn.edu.
1. Chun TW, et al. Quantification of latent tissue reservoirs and total body viral load in HIV-1 infection. Nature. 1997;387(6629):183-188.

2. Chun TW, Finzi D, Margolick J, Chadwick $\mathrm{K}$, Schwartz D, Siliciano RF. In vivo fate of HIV-1-infected T cells: quantitative analysis of the transition to stable latency. Nat Med. 1995;1(12):1284-1290.

3. Finzi D, et al. Identification of a reservoir for HIV-1 in patients on highly active antiretroviral therapy. Science. 1997;278(5341):1295-1300.

4. Hill AL, Rosenbloom DI, Fu F, Nowak MA, Siliciano RF. Predicting the outcomes of treatment to eradicate the latent reservoir for HIV-1. Proc Natl Acad Sci U S A . 2014;111(37):13475-13480.

5. Pinkevych $\mathrm{M}$, et al. Correction: HIV reactivation from latency after treatment interruption occurs on average every 5-8 days - implications for HIV remission. PLoS Pathog. 2016;12(8):e1005745.

6. Davey RT, et al. HIV-1 and T cell dynamics after interruption of highly active antiretroviral therapy (HAART) in patients with a history of sus- tained viral suppression. Proc Natl Acad Sci US A. 1999;96(26):15109-15114.

7. Chun TW, Davey RT, Engel D, Lane HC, Fauci AS. Re-emergence of HIV after stopping therapy. Nature. 1999;401(6756):874-875.

8. Li JZ, et al. The size of the expressed HIV reservoir predicts timing of viral rebound after treatment interruption. AIDS. 2016;30(3):343-353.

9. Rothenberger MK, et al. Large number of rebounding/founder HIV variants emerge from multifocal infection in lymphatic tissues after treatment interruption. Proc Natl Acad Sci U S A. 2015;112(10):E1126-E1134.

10. Finzi D, et al. Latent infection of $\mathrm{CD} 4^{+} \mathrm{T}$ cells provides a mechanism for lifelong persistence of HIV-1, even in patients on effective combination therapy. Nat Med.1999;5(5):512-517.

11. Siliciano JD, et al. Long-term follow-up studies confirm the stability of the latent reservoir for HIV-1 in resting CD $4^{+} \mathrm{T}$ cells. Nat Med. 2003;9(6):727-728.

12. Crooks AM, et al. Precise quantitation of the latent HIV-1 reservoir: Implications for eradication strategies. J Infect Dis. 2015;212(9):1361-1365.

13. Kutzler MA, Jacobson JM. Treatment interruption as a tool to measure changes in immunologic response to HIV-1. Curr Opin HIV AIDS. 2008;3(2):131-135.

14. Siliciano JD, Siliciano RF. Assays to measure latency, reservoirs, reactivation [Published online ahead of print October 26, 2017]. Curr Top Microbiol Immunol. https://doi.org/10.1007/82_2017_75.

15. Henrich TJ, et al. Antiretroviral-free HIV-1 remission and viral rebound after allogeneic stem cell transplantation: report of 2 cases. Ann Intern Med. 2014;161(5):319-327.

16. Strategies for Management of Antiretroviral Therapy (SMART) Study Group, et al. CD4 ${ }^{+}$ count-guided interruption of antiretroviral treatment. NEngl J Med. 2006;355(22):2283-2296.

17. Bar KJ, et al. Effect of HIV antibody VRCO1 on viral rebound after treatment interruption. $N$ Engl JMed. 2016;375(21):2037-2050.

18. Laird GM, Rosenbloom DI, Lai J, Siliciano RF, 
Siliciano JD. Measuring the frequency of latent HIV- 1 in resting $\mathrm{CD} 4^{+} \mathrm{T}$ cells using a limiting dilution coculture assay. Methods Mol Biol. 2016;1354:239-253.

19. Laskey SB, Pohlmeyer CW, Bruner KM, Siliciano RF. Evaluating clonal expansion of HIV-infected cells: optimization of PCR strategies to predict clonality. PLoS Pathog. 2016;12(8):e1005689.

20. Bui JK, et al. Proviruses with identical sequences comprise a large fraction of the replicationcompetent HIV reservoir. PLoS Pathog. 2017;13(3):e1006283.

21. Lee GQ, et al. Clonal expansion of genome-intact HIV-1 in functionally polarized Th1 $\mathrm{CD} 4^{+} \mathrm{T}$ cells. JClin Invest. 2017;127(7):2689-2696.

22. Hosmane NN, et al. Proliferation of latently infected $\mathrm{CD} 4^{+} \mathrm{T}$ cells carrying replication-competent HIV-1: potential role in latent reservoir dynamics. JExp Med. 2017;214(4):959-972.

23. Palmer S, et al. Multiple, linked human immunodeficiency virus type 1 drug resistance mutations in treatment-experienced patients are missed by standard genotype analysis. JClin Microbiol. 2005;43(1):406-413.

24. Mansky LM, Temin HM. Lower in vivo mutation rate of human immunodeficiency virus type 1 than that predicted from the fidelity of purified reverse transcriptase. J Virol. 1995;69(8):5087-5094.

25. Cohn LB, et al. HIV-1 integration landscape during latent and active infection. Cell. 2015;160(3):420-432.

26. Ho YC, et al. Replication-competent noninduced proviruses in the latent reservoir increase barrier to HIV-1 cure. Cell. 2013;155(3):540-551.

27. Bruner KM, et al. Defective proviruses rapidly accumulate during acute HIV-1 infection. Nat Med. 2016;22(9):1043-1049.

28. Cummings MP, Neel MC, Shaw KL. A genealogical approach to quantifying lineage divergence. Evolution. 2008;62(9):2411-2422.

29. Scheid JF, et al. HIV-1 antibody 3BNC117 suppresses viral rebound in humans during treatment interruption. Nature. 2016;535(7613):556-560.

30. Rosás-Umbert M, et al. Virological and immunological outcome of treatment interruption in HIV-1-infected subjects vaccinated with MVA-B. PLoS One. 2017;12(9):e0184929.

31. Blankson JN, et al. Biphasic decay of latently infected $\mathrm{CD} 4^{+} \mathrm{T}$ cells in acute human immunodeficiency virus type 1 infection. J Infect Dis. 2000;182(6):1636-1642.

32. Rosenbloom DIS, Hill AL, Laskey SB, Siliciano RF. Re-evaluating evolution in the HIV reservoir. Nature. 2017;551(7681):E6-E9.

33. Wang Z, et al. Expanded cellular clones carrying replication-competent HIV-1 persist, wax, and wane. Proc Natl Acad Sci U S A. 2018;115(11):E2575-E2584.

34. Jaafoura S, et al. Progressive contraction of the latent HIV reservoir around a core of lessdifferentiated $\mathrm{CD}^{+}$memory T cells. Nat Commun. 2014;5:5407.

35. Ananworanich J, et al. HIV DNA set point is rapidly established in acute HIV infection and dramatically reduced by early ART. EBioMedicine. 2016;11:68-72.

36. Ananworanich J, et al. Markers of HIV reservoir size and immune activation after treatment in acute HIV infection with and without raltegravir and maraviroc intensification. JVirus Erad.
2015;1(2):116-122.

37. Sneller MC, et al. A randomized controlled safety/efficacy trial of therapeutic vaccination in HIV-infected individuals who initiated antiretroviral therapy early in infection. Sci Transl Med. 2017;9(419):eaan8848.

38. Bailey JR, et al. Residual human immunodeficiency virus type 1 viremia in some patients on antiretroviral therapy is dominated by a small number of invariant clones rarely found in circulating $\mathrm{CD} 4^{+}$ T cells. J Virol. 2006;80(13):6441-6457.

39. Simonetti FR, et al. Clonally expanded CD $4^{+} \mathrm{T}$ cells can produce infectious HIV-1 in vivo. Proc Natl Acad Sci U S A. 2016;113(7):1883-1888.

40. Lorenzi JC, et al. Paired quantitative and qualitative assessment of the replication-competent HIV-1 reservoir and comparison with integrated proviral DNA. Proc Natl Acad Sci U S A. 2016;113(49):E7908-E7916.

41. Clarridge KE, et al. Effect of analytical treatment interruption and reinitiation of antiretroviral therapy on HIV reservoirs and immunologic parameters in infected individuals. PLoS Pathog. 2018;14(1):e1006792.

42. Cillo AR, et al. Improved single-copy assays for quantification of persistent HIV-1 viremia in patients on suppressive antiretroviral therapy. JClin Microbiol. 2014;52(11):3944-3951.

43. Palmer S, et al. New real-time reverse transcriptase-initiated PCR assay with single-copy sensitivity for human immunodeficiency virus type 1 RNA in plasma. JClin Microbiol.2003;41(10):4531-4536.

44. Laird GM, et al. Rapid quantification of the latent reservoir for HIV-1 using a viral outgrowth assay. PLoS Pathog. 2013;9(5):e1003398. 